\title{
A CASE REPORT OF A MALE PERSON OF 24 YEARS WITH SUSPECTED SALMONELLA INDUCED CORONARY ARTERITIS
}

\author{
DEB SR ${ }^{1}$, BARAL $^{2}$, MAKSUD ${ }^{3}$, KABIR A $^{4}$, HOSSAIN $\mathrm{M}^{5}$, HOSSAIN A $^{6}$
}

\begin{abstract}
:
Salmonella infection can cause a wide spectrum of disease which can range from a gastroenteritis, enteric fever (caused by typhoid and paratyphoid serotypes), bacteremia, focal infections, to a convalescent lifetime carrier state. Salmonella induced enteritis is common in this subcontinent. One third of untreated patients experience complications and account for three fourths of deaths associated with salmonellosis. But Salmonella induced coronary arteritis is rare and the initial diagnosis of those patients can be troublesome. Coronary arteritis may lead to fatal thrombosis, myocarditis, coronary artery aneurism, myocardial ischaemia, myocardial infarction $\&$ death. We reported a male person of 24 years who presented with high grade continued fever with rose spots, loose motion and vomiting and later on the tenth day of febrile illness he developed chest pain with subsequent ECG changes (ST elevation in V3-V5 chest leads) and elevated Troponin I level $(41.20 \mathrm{ng} / \mathrm{ml}$ ) which lead to the diagnosis of myocardial infarction.
\end{abstract}

J Dhaka Med Coll. 2014; 23(1) : 143-146.

\section{Introduction:}

Salmonella is a gram negative facultative anaerobic intracellular organism. The virulence of the organism depends on the subtype and host factors. Once the bacteria survive the acidic stomach, they colonize the intestine and translocate across the intestinal epithelium . After crossing this epithelial layer, the bacteria replicate in macrophages in Peyer's patches, mesenteric lymph nodes, and the spleen. Once colonized, the bacteria may then potentially disseminate to the lungs, gallbladder, kidneys, or central nervous system. The nontyphoid species of Salmonella tend to produce a more localized response because they are believed to lack the humanspecific virulence factors. However, the typhi serotype can develop the more invasive disease resulting in bacteremia. The severity of disease is related to the serotype, number of organisms, and host factors. Salmonella Typhi is estimated to outweigh Salmonella Paratyphi by a ratio of about 4:1 in endemic countries.
Untreated patients experience either complications or resolution by the fourth week. Intestinal perforation occurs in $3-10 \%$ of patients. Localized infection occurs in 5-10\% of persons with bacteremia. The endocardium, arteries, CNS (more commonly in infants), lungs, bones, joints, muscles, soft tissues, reticuloendothelial system, kidneys, and genital regions have all been documented sites of extraintestinal infection

\section{Case Report:}

A 24 years old male, university student, homosexual, muslim, hailing from Demra, Bangladesh presented to us on 03.03.13 with the complaints of high grade continued fever, not subsided by taking anti pyretics, not associated with chills \& rigor (highest recorded temperature was $104^{\circ} \mathrm{F}$ ) for last 5 days, non projectile, non bilious vomiting and loose motion for 8-10 episodes/day for the same duration, macular rashes at the same time in the back, chest, abdomen \& restlessness,

1. Dr.Sudip Ranjan Deb, Assistant Professor, Department of Medicine, Dhaka Medical College, Dhaka

2. Dr.Prianka Baral, Medical Officer, Department of Medicine, Dhaka Medical College, Dhaka

3. Dr.Maria Maksud, Medical Officer, Department of Medicine, Dhaka Medical College ,Dhaka

4. Dr.Ahmedul Kabir, Associate Professor, Department of Medicine, Dhaka Medical College, Dhaka

5. Dr.Mahbub Hossain,Indoor Medical officer, Department of Medicine,Dhaka Medical College, Dhaka

6. Dr. Anwar Hossain, Assistant Registrar, Department of Medicine, Dhaka Medical College, Dhaka

Correspondence: Dr.Sudip Ranjan Deb, Assistant Proffessor, Department of Medicine, Dhaka Medical College, Dhaka,Cell Phone :+8801714963196 Email:sudipranjandev@yahoo.com 
confusion \& irrelevant talking for last 1 day. His serum electrolyte report reveals severe hyponatremia (S.Na-106 meq/1).This was corrected with $3 \% \mathrm{NaCl}$ solution. The patient was being treated with inj. Ceftriaxone (2 gram) 12 hourly suspecting Enteric fever. Then on the seventh day of febrile illness, he complained of passage of blood mixed stool which was soft , voluminous \& sticky four to five times a day. He also complained of generalised bodyache. On the tenth day of fever , he complained of central chest pain. His 12 lead ECG was done $\&$ it reveals ST elevation in V3,V4,V5 leads. ECHO shows no regional hypokinesia, ejection fraction was $66 \%$. Cardiac Troponin -I level was highly elevated(41.20 ng/ml. He did not receive Inj. Streptokinase due to per rectal bleeding. On the 12 th day, he stopped passing blood mixed stool but developed loose stool for 15-20 times a day. On the fifteenth day, he developed psychosis, talking irrelevantly \& attempting to bite the people around him. On query, he gave no history of yellow coloration of urine, hematemesis, passage of black tarry stool, bleeding through any other route, no history of recent travel history or any kind of drug abuse. There is no history of cardiac disease in his family.

On examination, he was ill-looking, body build average, non-anaemic, non icteric , non oedematous, moderately dehydrated. Skin Condition- macular rashes present on back, abdomen, chest. Lymphadenopathy-absent, Blood Pressure-90/60 mmHg , Pulse-120/min,

Respiratory rate-22 breaths / min, Temp.-102.5 ${ }^{0} \mathrm{~F}$. On alimentary system examination, tongue was coated, superficial palpation-abdomen was tender, more on right \& left hypochondriac region, Liver was non-palpable, spleen-initially was palpable, $3 \mathrm{~cm}$ from the subcostal margin along its axis towards the right iliac fossa, soft, tender, later disappear during the course of treatment. shifting dullness absent, other systemic examination revealed no abnormality .Digital rectal examination- There was unhealthy rectal mucosa. No growth was found. Finger was stained by fecal matter. ProctoscopyThere was no significant findings. Multiple erosion were present on rectal mucosa.
His investigations revealed: Complete Blood Count: HB- $12.0 \mathrm{~g} / \mathrm{dl}$, Total count $6330 / \mathrm{mm} 3$, Neutrofil-66.5\%, Lymphocyte-23.4\%. Peripheral blood film- RBC- Normocytic normochromic ,WBC-Mature with above count $\&$ distribution. Platelet -Normal, ESR-49 $\mathrm{mm}$ in $1^{\text {st }}$ hour. S. electrolytes- $\mathrm{Na}+106 \mathrm{mmol} / \mathrm{l}, \mathrm{K}+3.40$ mmol/1. Repeat S. electrolytes: $\mathrm{Na}+137.0$ $\mathrm{mmol} / \mathrm{L} \quad \mathrm{K}+3.7 \mathrm{mmol} / \mathrm{l}$. PBF \& ICT for malaria-negative, S. Creatinine- $0.6 \mathrm{mg} / \mathrm{dl}$, Urine R/M/E- Normal finding .E.C.G. - ST elevation in V3,V4,V5 leads, Echocardiogram shows- No regional wall motion akinesia, Good LV Systolic Function .Cardiac Troponin I- $41.20 \mathrm{ng} / \mathrm{ml}$, Repeat Echo- septal, anterior lateral wall is mildly hypokinetic with EF47\%.Coronary Angiogram shows Normal Coronary arteries, ICT for Dengue AntibodiesIg $\mathrm{G}$ - negative, Ig $\mathrm{M}$ - negative , $\mathrm{D}$-dimer- 0.48 $\mathrm{mg} / 1$ (normal $<0.3 \mathrm{mg} / 1$ ), Plasma Fibrin degradation product- $14.9 \mathrm{Ug} / \mathrm{ml}$, Ultrasonogram of whole abdomen)- Normal Findings, Blood culture(FAN method)- No Growth, ANA-Negative, Anti phospholipid AbIg M \& Ig G-Negative, Febrile Antigen: Widal Test: Negative, Rickettsial Antibody: Negative , Brucella Abortus Antibody: $<1: 80$, Brucella Maletensis antibody: <1:80.Fasting Lipid Profile: normal findings, S.Bilirubin- $0.66 \mathrm{mg} /$ d1, SGOT-20.0 U/1,SGPT-88.0 U/1 ,HBsAgnegative, HIV (1 \& 2)- negative, VDRL- nonreactive, Anti HCV Ab- negative. Endoscopy \& colonoscopy -Normal findings.

\section{Discussion:}

Salmonellae are gram-negative, non-sporeforming, facultative anaerobic bacilli. Foodborne or waterborne transmission occurs as a result of fecal contamination by ill or asymptomatic chronic carriers. Usually, waterborne transmission involves the ingestion of fewer microorganisms and, as a result, has a longer incubation period and lower attack rate compared with foodborne transmission. Although direct person-to-person transmission is uncommon, S. Typhi can be transmitted sexually, including by anal and oral sex. ${ }^{1}$ Health care workers can acquire the disease from infected patients as a result of poor hand hygiene or handling laboratory specimens. 
Enteric fever continues to be a global health problem, with an estimated 21.6 million cases caused by $S$. Typhi and 5.5 million cases

caused by S. Paratyphi A, B, or C annually and an incidence ranging from 25 to 1000 cases per 100,000 population in endemic regions. ${ }^{2,3}$

An estimated 200,000 to 600,000 deaths occur annually, based on extrapolation from endemic regions. ${ }^{2}$ Regions with a high incidence of typhoid fever (>100/100,000 cases/year) include south-central Asia and southeast Asia. Regions of medium incidence (10-100/100,000 cases/year) include the rest of Asia, Africa, Latin America and the Caribbean, and Oceania, except for Australia and New Zealand .$^{2}$ The incidence of enteric fever correlates with poor sanitation and lack of access to clean drinking water. In endemic regions, typhoid fever is more common in urban than rural areas and among young children and adolescents (aged 1 to 15 years).

Reported risk factors include contaminated water or ice, flooding, food and drinks purchased from street vendors, raw fruits and vegetables grown in fields fertilized with sewage, ill contacts in the household, lack of hand washing and toilets, and evidence of prior Helicobacter pylori infection, likely related to chronic reduced gastric acidity. Outbreaks of typhoid fever in developing countries can result in high morbidity and mortality, especially among children less than 5 years of age and when caused by antimicrobial-resistant strains. ${ }^{4,5}$ Between 1970 and 1989, many strains of $S$. Typhi developed plasmid-mediated multidrug resistance to the common first-line antimicrobials chloramphenicol, ampicillin, and trimethoprim in many regions of the world, especially in the Indian subcontinent and south Asia. ${ }^{6}$

Up to $8 \%$ of patients with nontyphoidal Salmonellagastroenteritis develop bacteremia; of these, $5 \%$ to $10 \%$ develop localized infections. Bacteremia and metastatic infection are more common with $S$. Choleraesuis and S. Dublin and among infants, the elderly, and those who are immunocompromised. ${ }^{7}$ Among children, nontyphoidal
Salmonella bacteremia usually is associated with gastroenteritis and prolonged fever, infrequently causes focal infections, and is fatal in less than $10 \%$ of cases. ${ }^{8}$ In contrast, adults are more likely to have primary bacteremia and have a high incidence of secondary focal infections and death. ${ }^{8}$ The mortality of nontyphoidal Salmonella bacteremia increases with the magnitude of bacteremia and in the presence of coma or septic shock. ${ }^{9}$

Salmonella have a propensity for infection of vascular sites, including vascular grafts, and high-grade or persistent bacteremia suggests an endovascular infection. ${ }^{10}$ The risk of endovascular infection complicating Salmonella bacteremia is estimated to be $9 \%$ to $25 \%$ in persons over 50 years of age, usually involves the aorta, and most commonly results from seeding atherosclerotic plaques or aneurysms. ${ }^{11}$ In endovascular Salmonella infection resulting in true (or false) aneurysms, involvement such as the thoracic and abdominal aorta, ${ }^{16}$ coronary arteries ${ }^{14,15}$ and peripheral arteries ${ }^{17}$ have all been documented. Arteriovenous fistulae can also result. Mortality rates range from $14 \%$ to $60 \%$ and are lower with prompt diagnosis and combined medical and surgical therapy. ${ }^{8}$ Venous septic thrombophlebitis also has been reported. ${ }^{12}$

In a series of patients with bacteremia due to Salmonella, $25 \%$ of those older than 50 years developed an endothelial infection ${ }^{13}$. This reflects the ability of Salmonella, which has been reported to invade normal arterial intima, to cause endothelial infection in the presence of atherosclerosis ${ }^{13}$. The predominance of older patients with or without hypertension among patients with Salmonella aortitis is probably due to the increased incidence of atherosclerosis and intimal damage in these patients. Recently, Salmonella bacteremia has been noted in patients with human immunodeficiency virus infection; however, aortitis rarely occurs in these patients because they are relatively younger and do not have the above risk factors. 
We report this case because our patient presented to us in a confused state $\&$ was clinically suspected $\&$ treated as a case of Enteric Fever caused by Salmonella Typhi although Blood culture in FAN Method revealed no growth. But the patient developed high grade continued fever pattern (highest $104^{\prime} \mathrm{F}$ ), vomiting, diarrhea, abdominal cramp, rose spots, splenomegaly \& electrolyte imbalance (Hyponatremia) which direct to the diagnosis of Enteric Fever. As he is a homosexual person the possibility of transmission of salmonella typhi infection through anal sex shoud be considered cautiously. But Salmonella induced Coronary arteritis is found in immunocompromised patient or in HIV patient or with Non-typhoidal Salmonella subtypes. Our patient was not found to be immunocompromised. No cardiovascular risk factor was present. No family history of cardiac disease was present .Although he responded well to Inj. Ceftriaxone (2 gm) B.D, on the Tenth day of febrile illness he developed chest pain. ECG revealed ST elevation in V3-V6 Chest Leads \& highly elevated Troponin I level(41.20 $\mathrm{ng} / \mathrm{ml})$. Subsequent Coronary Angiogram revealed normal coronary arteries. Initially Echo was normal but later on Echo shows septal, anterior lateral wall is mildly hypokinetic with EF- 47\%.

Now the patient is well after receiving 6 weeks of Inj. Ceftriaxone and now he is on Tab. Aspirin (75mg) and Tab. Carvedilol (6.25mg) daily.

\section{Referrences:}

1. Reller ME, Olsen SJ, Kressel AB, et al. Sexual transmission of typhoid fever: a multistate outbreak among men who have sex with men. Clin Infect Dis. 2003; 37:141-144.

2. Crump JA, Luby SP, Mintz ED. The global burden of typhoid fever. Bull World Health Organ. 2004; 82:346-353.

3. Ochiai RL, Acosta CJ, Danovaro-Holliday MC, et al. A study of typhoid fever in five Asian countries: disease burden and implications for controls. Bull World Health Organ. 2008; 86: 260-268.
4. Sinha A, Sazawal S, Kumar R, et al. Typhoid fever in children aged less than 5 years. Lancet. 1999; 354: 734-737.

5. Bhutta ZA, Naqvi SH, Razzaq RA, et al. Multidrugresistant typhoid in children: presentation and clinical features. Rev Infect Dis. 1991; 13: 832-836.

6. Rowe B, Ward LR, Threlfall EJ. Multidrugresistant Salmonella Typhi: a worldwide epidemic. Clin Infect Dis. 1997; 24(Suppl 1): S106-S109.

7. Han T, Sokal JE, Neter E. Salmonellosis in disseminated malignant diseases: a seven-year review (1959-1965). N Engl J Med. 1967;276:10451052 .

8. Shimoni Z, Pitlik S, Leibovici L, et al. Nontyphoid Salmonella bacteremia: age-related differences in clinical presentation, bacteriology, and outcome. Clin Infect Dis. 1999; 28: 822-827.

9. Parsons R, Gregory J, Palmer DL. Salmonella infections of the abdominal aorta. Rev Infect Dis. 1983; 5: 227-231.

10. Cohen JI, Bartlett JA, Corey GR. Extra-intestinal manifestations of Salmonella infections. Medicine. 1987; 66: 349-388.

11. Carey J, Buchstein S, Shah S. Septic deep vein thrombosis due to Salmonella johannesburg. $J$ Infect. 2001; 42: 79-80.

12. Cooke FJ, Day M, Wain J, et al. Cases of typhoid fever imported into England, Scotland and Wales (2000-2003). Trans R Soc Trop Med Hyg. 2007; 101 : 398-404.

13. Barnett, R.N. \& Zimmerman, S.L. Coronary arteritis with fatal thrombosis due to Salmonella choleraesuis variety kunzendorf. Am Heart J 1947, 34: 441-446.

14. McGee, M.B. \& Khan, M.Y. Ruptured mycotic aneurysm of a coronary artery: A fatal complication of salmonella infection. Arch Intern Med 1980, 140: 1097-1098.

15. Parsons, R., Gregory, J. \& Palmer, D. Salmonella infections of the abdominal aorta. Rev Infect Dis 1983, 5: 227-231.

16. Rubin, R.H. \& Weinstein, L. Salmonella Microbiologic, Pathologic and Clinical Features. Stratton Intercontinental Medical Book Corp, New York, 1977. 